\title{
新型含三唑啉酮的磺酰腿类化合物的合成、晶体结构及除草活性研究
}

\author{
潘里陈有为刘卓李永红李正名* \\ (南开大学元素有机化学研究所 元素有机化学国家重点实验室 天津 300071)
}

\begin{abstract}
摘要 为了进一步寻找高效的磺酰服类除草剂, 以商品化磺酰腿类除草剂为基础, 将三唑啉酮杂环引入到分子中, 合 成了 23 个未见文献报道的新型磺酰脉化合物，通过 ${ }^{1} \mathrm{H} N \mathrm{NR}$ 、高分辨质谱以及 $\mathrm{X}$ 射线单晶衍射确定了其结构. 经盆栽 法和平皿法测试了所有化合物的除草活性以及部分化合物对油菜的 $\mathrm{IC}_{50}$ 值. 结果表明, 化合物 $\mathbf{5 h}$ 具有优秀的除草活性, 其对油菜的 $\mathrm{IC}_{50}$ 值与对照药醚苯磺隆和梄磺隆相近, 在 $3.75 \mathrm{~g} / \mathrm{ha}$ 浓度下对单子叶杂草稗草具有优异的盆栽抑制活性. 关键词＼cjkstart磺酰艰; 三唑啉酮; 合成; 晶体结构; 除草活性
\end{abstract}

\section{Synthesis, Crystal Structure and Herbicidal Activity of Novel Sulfonylureas Containing Triazolinone Moiety}

\author{
Pan, Li Chen, Youwei Liu, Zhuo Li, Yonghong Li, Zhengming* \\ (Research Institute of Elemento-organic Chemistry, State Key Laboratory of Elemento-organic Chemistry, \\ Nankai University, Tianjin 300071)
}

\begin{abstract}
In order to search for efficient sulfonylurea herbicides, the title compounds were designed by introducing triazolinones into some known sulfonylurea structures. 23 novel sulfonylurea compounds had been synthesized and characterized by ${ }^{1} \mathrm{H}$ NMR, HRMS and X-ray diffraction. Herbicidal activities of title compounds were determined by pot-culture method. $\mathrm{IC}_{50}$ values of some effective sulfonylurea compounds were tested by culture dish method. It was found that compound $\mathbf{5} \mathbf{h}$ attained the super-active level which was comparable to the controls as triasulfuron and cinosulfuron. It was worthy to note that compound $\mathbf{5 h}$ had potent inhibitory activity against Echinochloa crusgalli by pot-culture method at $3.75 \mathrm{~g} / \mathrm{ha}$.

Keywords sulfonylurea; triazolinone; synthesis; crystal structure; herbicidal activity
\end{abstract}

乙酰羟基酸合成酶(AHAS)也称乙酰乳酸合成酶 (ALS)，它是高等植物体内支链氨基酸——线氨酸、亮氨 酸和异亮氨酸生物合成第一步的催化酶 ${ }^{[1]}$, 能催化一分 子丙酮酸脱羧并与另一分子丙酮酸缩合成一分子乙酰 乳酸. 由于高等动物自身不能合成这些支链氨基酸，需 要从外界食物中摄取, 因此该酶是理想的除草剂作用靶 点 ${ }^{[25]}$. 磺酰脲是以 AHAS 酶为靶标的一类重要除草 剂 $^{[6,7]}$, 从而使得磺酰脲类除草剂对哺乳动物具有很低 的毒性. 正因为这个特点, 磺酰脲类除草剂得到了广泛 应用 ${ }^{[8 \sim 10]}$.

杂环化合物 ${ }^{[1]}$ 由于其多变的结构和广泛的生物活 性使得在农药的开发中受到广泛的关注, 尤其是三唑类
含氮杂环 ${ }^{[12 ~ 14]}$. 三唑啉酮类化合物因具有很好的除草 活性 ${ }^{[15 ~ 18]}$ 已成为农药创制中的热点. 自从杜邦公司发 现了第一个三唑啉酮类除草剂唑啶草酮后, 很多三唑啉 酮类除草剂相继被开发出来, 例如: 氨唑草酮、甲磺草 胺、唑草酮等. 同时, 近年来出现的氟酩磺隆、丙苯磺 隆也具有三唑啉酮结构. 因此新三唑啉酮类化合物具有 很好的研究前景.

综上所述，我们将三唑啉酮引入到已知商品化除草 剂(如醚苯磺隆、醚磺隆等)分子中, 设计出结构新颖的 含三唑啉酮的苯基磺酰艮或杂环磺酰䐂结构，以期得到 除草活性更好的磺酰脲类化合物. 目标化合物合成路线 见 Scheme 1 .

\footnotetext{
* E-mail: nkzml@vip.163.com
}

Received November 8, 2012; revised December 12, 2012; published online December 18, 2012

Project supported by the National Natural Science Foundation of China (No. 21272129), the National Basic Research Program of China (No. 2010CB126106) and the National Key Technologies R\&D Program (No. 2011BAE06B05).

国家自然科学基金(No. 21272129)、国家重点基础研究发展计划(973, No. 2010CB126106)、国家科技攻关计划(No. 2011BAE06B05)资助项目. 


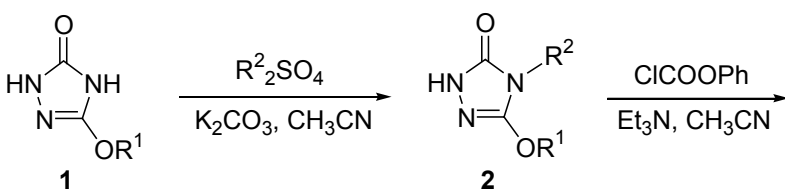<smiles>[R]Oc1nn(C(=O)O[Na])c(=O)n1[R]</smiles>

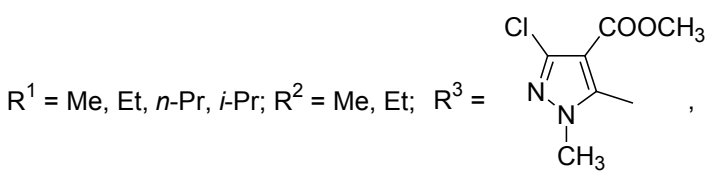<smiles></smiles>

Scheme 1

\section{1 结果与讨论}

\section{1 合成讨论}

在三唑甲酸苯酯 3 的合成中, 曾尝试使用氢氧化 钠、碳酸钾、三乙胺、吡啶等碱作为缚酸剂, 但是除三 乙胺以外, 其他几种缚酸剂在反应中均得不到较好的收 率, 所以将此反应的缚酸剂确定为三乙胺, 这可能与三 乙胺碱性适中有关, 同时过量的三乙胺可以通过旋转蒸 发的方式除去, 不会残留在体系中. 同时在合成 3 的过 程中, 为了防止反应过于剧烈, 用乙腈将氯甲酸苯酯稀 释再滴入到反应体系中, 然而仅仅得到少量目标产物, 大多数为氯甲酸苯酯缩合的副产物一一碳酸二苯酯. 当 用纯的氯甲酸苯酯滴加时, 时间应控制在 $20 \sim 30 \mathrm{~min}$ 内, 如果滴加时间过短会导致反应剧烈放热，从而使反 应失败, 过长则会使氯甲酸苯酯缩合成为碳酸二苯酯而 影响目标产物的收率.

在目标化合物 $\mathbf{5}$ 的合成中, 曾尝试使用三乙胺作为 催化剂, 但是用于碱性不够强, 从而导致缩合反应不能 彻底进行，降低产品收率．因此换用常用有机强碱一 DBU 作为反应催化剂, 使得反应的收率得到较大提高.

\section{2 晶体结构}

晶体结构分析表明(图 1): 证明了三唑啉酮环中 1 位的氮原子与嫝桥上的羰基相连, 4 位的氮原子与烷基 相连. 吡唑环、磺酰基、酰胺基、酯基的键长键角都与 文献[19 21]报道的一致. $\mathrm{C}(2)-\mathrm{N}(2)-\mathrm{C}(1)-\mathrm{O}(1)$ 之间 二面角为 $-176.6(2)^{\circ}, \mathrm{N}(3)-\mathrm{N}(2)-\mathrm{C}(1)-\mathrm{O}(1)$ 之间二
面角为 $-0.8(3)^{\circ}$, 说明了羰基与三唑啉环基本在一个平 面上，存在比较强的共轭作用。另外 $\mathrm{C}(6)-\mathrm{C}(8)-$ $\mathrm{C}(10)-\mathrm{O}(6)$ 之间的二面角为 $10.4(4)^{\circ}, \mathrm{C}(7)-\mathrm{C}(8)-$ $\mathrm{C}(10)-\mathrm{O}(6)$ 之间的二面角为 $-167.8(2)^{\circ}$, 也说明羰基与 吡唑环基本在同一平面. 三唑啉酮 $[\mathrm{N}(2)-\mathrm{N}(3)-\mathrm{C}(3)-$ $\mathrm{N}(4)-\mathrm{C}(2)$ ] 的五元环为一个平面, 与吡唑环平面 $[\mathrm{N}(5)-\mathrm{N}(6)-\mathrm{C}(7)-\mathrm{C}(8)-\mathrm{C}(6)]$ 形成的二面角为 $93.5^{\circ}$, 几乎垂直.嫝桥上 $\mathrm{N} 1$ 相连的 $\mathrm{H} 1$ 与三唑啉酮羰基上的 $\mathrm{O} 2$ 形成了氢键作用; 同时三唑酮的甲基的 $\mathrm{C}-\mathrm{H}$ 与吡唑环 存在 $\mathrm{C}-\mathrm{H}-\pi$ 的左右, 其距离为 $3.547 \AA$, 这对整个分子 起到了稳定作用.

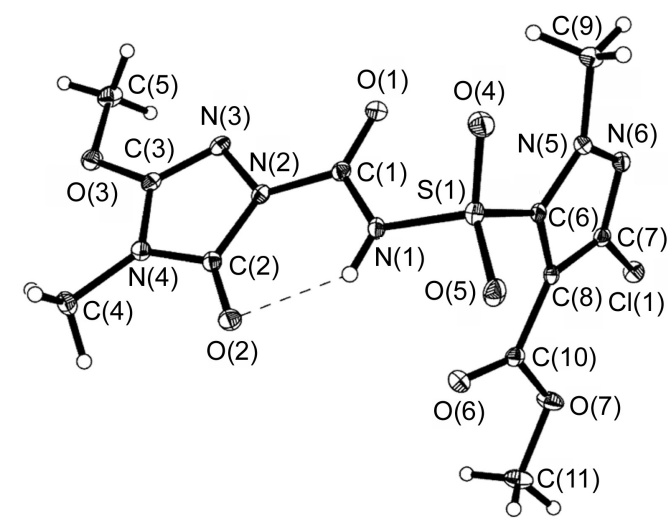

图 1 化合物 $5 \mathrm{a}$ 的晶体结构图

Figure 1 Crystal structure of compound 5a

\section{3 生物活性}

目标化合物 5 对双子叶杂草油菜(Brassica napus)和 反枝苋(Amaranthus retroflexus) 及单子叶杂草稗草 (Echinochloa crusgalli)和马唐(Digitaria adscendens)的生 物活性测定结果如表 $1 \sim 3$ 所示.

从表 1 的 $375 \mathrm{~g} / \mathrm{ha}$ 剂量的初篎结果可以看出, 大部 分化合物对双子叶作物的除草活性明显高于单子叶作 物. 当 $\mathrm{R}^{3}$ 是 2-(甲氧基乙氧基)苯基或 2-(氯乙氧基)苯基 时, 除草活性优于其它取代基. $\mathrm{R}^{2}$ 是甲基的化合物普遍 除草活性高于乙基. 表 2 的降低浓度复篎结果表明，大 部分化合物在降低浓度后，活性有所下降. 但 $\mathbf{5 h}$ 在浓度 变成初笁的 $1 / 100$ 后, 对油菜仍具有良好的除草活性, 抑制率高于对照药醚磺隆, 与醚苯磺隆和单嘧磺酯相 当. 同时 $5 \mathbf{h}$ 在 $3.75 \mathrm{~g} / \mathrm{ha}$ 浓度下对稗草的茎叶处理抑制 活性也在 $90 \%$ 以上，优于三种对照药的活性. 化合物 $\mathbf{5 j}$ 在 $15 \mathrm{~g} / \mathrm{ha}$ 的浓度下, 对双子叶杂草具有一定的选择性 (对油菜具有良好的活性，而对反枝苋则没有活性), 此 化合物值得进一步进行结构改造, 寻找具有选择性更好 的磺酰脲类除草剂. 我们对一些降低浓度后仍具有一定 除草活性的化合物进行了 $\mathrm{IC}_{50}$ 值测定(表 3), 结果显示 $5 \mathbf{h}$ 具有和商品化磺酰嫝除草剂醚磺隆、醚苯磺隆相近的 
表 1 目标化合物 5 在 $375 \mathrm{~g} / \mathrm{ha}$ 剂量下对杂草的抑制率(盆栽法)(抑制率/\%)

Table 1 Inhibitory rate (\%) of the target compounds 5 by pot-culture method against herbs at $375 \mathrm{~g} / \mathrm{ha}$

\begin{tabular}{|c|c|c|c|c|c|c|c|c|}
\hline \multirow{2}{*}{ Compd. } & \multicolumn{2}{|c|}{ Brassica napus } & \multicolumn{2}{|c|}{ Amaranthus retroflexus } & \multicolumn{2}{|c|}{ Echinochloa crusgalli } & \multicolumn{2}{|c|}{ Digitaria adscendens } \\
\hline & Soil treatment & Foliage spray & Soil treatment $\mathrm{I}$ & Foliage spray & Soil treatment & Foliage spray & Soil treatment $\mathrm{F}$ & Foliage spray \\
\hline $5 a$ & 0.0 & 0.0 & 0.0 & 15.0 & 0.0 & 10.0 & 0.0 & 0.0 \\
\hline $5 \mathbf{b}$ & 0.0 & 0.0 & 29.4 & 53.4 & 0.0 & 0.0 & 0.0 & 15.0 \\
\hline $5 c$ & 0.0 & 0.0 & 0.0 & 15.0 & 0.0 & 10.0 & 35.0 & 15.0 \\
\hline 5d & 0.0 & 0.0 & 0.0 & 52.8 & 0.0 & 0.0 & 40.0 & 10.0 \\
\hline $5 e$ & 0.0 & 0.0 & 45.9 & 15.0 & 0.0 & 15.0 & 10.0 & 10.0 \\
\hline $5 f$ & 100 & 100 & 100 & 100 & 100 & 100 & 100 & 100 \\
\hline $5 g$ & 90.8 & 100 & 92.9 & 100 & 45.1 & 100 & 100 & 100 \\
\hline $5 \mathrm{~h}$ & 93.3 & 100 & 97.2 & 100 & 77.3 & 100 & 78.0 & 67.9 \\
\hline $5 i$ & 95.0 & 100 & 99.1 & 100 & 69.1 & 73.0 & 65.0 & 75.0 \\
\hline $5 \mathbf{j}$ & 95.0 & 100 & 92.6 & 100 & 73.2 & 82.8 & 35.0 & 70.0 \\
\hline $5 \mathrm{k}$ & 78.7 & 100 & 94.4 & 95.0 & 59.8 & 95.0 & 30.0 & 35.0 \\
\hline 51 & 0.0 & 20.0 & 0.0 & 29.1 & 0.0 & 0.0 & 10.0 & 5.0 \\
\hline $5 \mathrm{~m}$ & 0.0 & 40.0 & 0.0 & 23.0 & 0.0 & 5.0 & 30.0 & 5.0 \\
\hline $5 n$ & 15.0 & 15.0 & 0.0 & 5.0 & 10.0 & 0.0 & 15.0 & 0.0 \\
\hline 50 & 0.0 & 5.0 & 0.0 & 10.0 & 0.0 & 10.0 & 10.0 & 5.0 \\
\hline $5 p$ & 86.6 & 100 & 95.4 & 97.0 & 64.9 & 82.8 & 60.0 & 46.2 \\
\hline $5 q$ & 90.5 & 100 & 94.4 & 100 & 41.2 & 97.0 & 60.0 & 10.0 \\
\hline $5 r$ & 83.8 & 100 & 98.1 & 100 & 74.2 & 100 & 77.0 & 70.0 \\
\hline $5 s$ & 10.0 & 89.5 & 0.0 & 90.0 & 26.8 & 56.5 & 15.0 & 34.6 \\
\hline $5 t$ & 93.8 & 100 & 98.1 & 100 & 73.2 & 100 & 75.0 & 65.0 \\
\hline $5 u$ & 81.0 & 100 & 93.5 & 99.0 & 34.0 & 81.3 & 10.0 & 40.0 \\
\hline $5 v$ & 78.2 & 100 & 61.1 & 55.0 & 19.6 & 72.3 & 15.0 & 5.0 \\
\hline $5 w$ & 29.5 & 100 & 76.9 & 85.0 & 10.0 & 10.0 & 10.0 & 25.0 \\
\hline Monosulfuron-ester & 100 & 100 & 100 & 100 & 100 & 100 & 100 & 100 \\
\hline Triasulfuron & 100 & 100 & 100 & 100 & 100 & 100 & 100 & 100 \\
\hline Cinosulfuron & 100 & 100 & 100 & 100 & 100 & 100 & 100 & 100 \\
\hline
\end{tabular}

表 2 部分目标化合物在 $15,7.5$ 和 $3.75 \mathrm{~g} / \mathrm{ha}$ 剂量下对杂草的抑制率(盆栽法)(抑制率/\%)

Table 2 Inhibitory rate (\%) of some target compounds by pot-culture method against herbs at 15, 7.5 and $3.75 \mathrm{~g} / \mathrm{ha}$

\begin{tabular}{|c|c|c|c|c|c|c|}
\hline \multirow{2}{*}{ Compd. } & \multirow{2}{*}{ Dosage $/\left(\mathrm{g} \cdot \mathrm{ha}{ }^{-1}\right)$} & \multicolumn{2}{|c|}{ Brassica napus } & \multicolumn{2}{|c|}{ Amaranthus retroflexus } & \multirow{2}{*}{$\begin{array}{c}\text { Echinochloa crusgalli } \\
\text { Foliage spray } \\
\end{array}$} \\
\hline & & Soil treatment & Foliage spray & Soil treatment & Foliage spray & \\
\hline \multirow{3}{*}{$5 f$} & 15 & 100 & 100 & 99.4 & 80.7 & 97.1 \\
\hline & 7.5 & 85.6 & 65.9 & 61.0 & 55.4 & 66.0 \\
\hline & 3.75 & 41.3 & 29.8 & 34.8 & 18.1 & 36.4 \\
\hline \multirow{3}{*}{$5 \mathrm{~g}$} & 15 & 20.0 & 100 & 0.0 & 22.8 & 0.0 \\
\hline & 7.5 & - & 34.1 & - & - & - \\
\hline & 3.75 & - & 21.9 & - & - & - \\
\hline \multirow{3}{*}{$5 \mathbf{h}$} & 15 & 98.3 & 94.1 & 77.2 & 22.2 & 100 \\
\hline & 7.5 & 94.8 & 100 & 76.6 & - & 100 \\
\hline & 3.75 & 91.5 & 92.0 & 28.7 & - & 90.1 \\
\hline \multirow{3}{*}{$5 \mathbf{i}$} & 15 & 71.1 & 77.4 & 25.1 & 12.3 & 0.0 \\
\hline & 7.5 & 60.5 & 23.7 & - & - & - \\
\hline & 3.75 & 30.3 & 0.0 & - & - & - \\
\hline \multirow{3}{*}{$5 \mathbf{j}$} & 15 & 92.7 & 73.2 & 0.0 & 0.0 & 0.0 \\
\hline & 7.5 & 77.5 & 31.8 & - & - & - \\
\hline & 3.75 & 49.1 & 0.0 & - & - & - \\
\hline
\end{tabular}




\begin{tabular}{|c|c|c|c|c|c|c|}
\hline \multirow{2}{*}{ Compd. } & \multirow{2}{*}{ Dosage/(g•ha $\left.{ }^{-1}\right)$} & \multicolumn{2}{|c|}{ Brassica napus } & \multicolumn{2}{|c|}{ Amaranthus retroflexus } & \multirow{2}{*}{$\begin{array}{c}\text { Echinochloa crusgall } \\
\text { Foliage spray }\end{array}$} \\
\hline & & Soil treatment & Foliage spray & Soil treatment & Foliage spray & \\
\hline \multirow{3}{*}{$5 \mathbf{k}$} & 15 & 0.0 & 63.1 & 0.0 & 0.0 & 21.2 \\
\hline & 7.5 & - & 42.0 & - & - & - \\
\hline & 3.75 & - & 12.5 & - & - & - \\
\hline \multirow{3}{*}{$5 p$} & 15 & 0.0 & 80.7 & 0.0 & 0.0 & 0.0 \\
\hline & 7.5 & - & 70.5 & - & - & - \\
\hline & 3.75 & - & 9.1 & - & - & - \\
\hline \multirow{3}{*}{$5 q$} & 15 & 66.5 & 19.1 & 21.5 & 12.3 & 5.5 \\
\hline & 7.5 & 64.9 & - & - & - & - \\
\hline & 3.75 & 57.9 & - & - & - & - \\
\hline \multirow{3}{*}{$5 t$} & 15 & 81.7 & 74.8 & 16.9 & 29.2 & 21.2 \\
\hline & 7.5 & 69.7 & 37.8 & - & - & - \\
\hline & 3.75 & 62.4 & 26.0 & - & - & - \\
\hline \multirow{2}{*}{ Monosulfuron-ester } & 7.5 & 96.3 & 100 & 86.1 & 53.4 & 23.5 \\
\hline & 3.75 & 81.2 & 92.6 & 27.6 & 26.5 & 16.7 \\
\hline \multirow{2}{*}{ Triasulfuron } & 7.5 & 100 & 100 & 100 & 83.6 & 46.9 \\
\hline & 3.75 & 98.2 & 100 & 94.4 & 29.4 & 0.0 \\
\hline \multirow{2}{*}{ Cinosulfuron } & 7.5 & 88.2 & 56.8 & 99.4 & 48.0 & 29.6 \\
\hline & 3.75 & 79.7 & 52.8 & 75.5 & 27.0 & 8.6 \\
\hline
\end{tabular}

表 3 部分目标化合物对油菜的 $\mathrm{IC}_{50}$ 值(平㿼法)

Table $3 \mathrm{IC}_{50}$ values of some target compounds by culture dish method against Brassica napus

\begin{tabular}{cccc}
\hline Compd. & $\mathrm{IC}_{50} /\left(\mathrm{mg} \cdot \mathrm{L}^{-1}\right)$ & Compd. & $\mathrm{IC}_{50} /\left(\mathrm{mg} \cdot \mathrm{L}^{-1}\right)$ \\
\hline $\mathbf{5 f}$ & 0.1019 & $\mathbf{5 p}$ & 0.3040 \\
$\mathbf{5 g}$ & 1.3380 & $\mathbf{5 q}$ & 0.8442 \\
$\mathbf{5 h}$ & 0.0761 & $\mathbf{5 t}$ & 0.3516 \\
$\mathbf{5 i}$ & 0.4473 & Monosulfuron-ester & 0.0078 \\
$\mathbf{5 j}$ & 0.2649 & Triasulfuron & 0.0102 \\
$\mathbf{5 k}$ & 0.9837 & Cinosulfuron & 0.0265 \\
\hline
\end{tabular}

超高效除草活性, 此化合物可做为结构进一步优化的先 导结构.

\section{2 结论}

合成了 23 个含有三唑啉酮结构的新型磺酰嫝类化 合物, 发现部分化合物具有良好的除草活性以及选择 性. 其中化合物 $5 \mathbf{h}$ 对双子叶杂草油菜具有与商品化磺 酰脲除草剂相当的抑制活性，对单子叶杂草稗草的抑制 活性高于商品化对照药. 因此, 化合物 $\mathbf{5 h}$ 可以做为先导 化合物进一步进行结构优化, 寻找活性更好的磺酰嫝类 除草剂.

\section{3 实验部分}

\section{1 仪器与试剂}

X-4 数字显示显微熔点测定仪(北京泰克仪器有限
公司); FTICR-MS (Varian 7.0T) 型高分辨率质谱仪; Bruker Avance-300 MHz 和 Bruker Avance-400 MHz 核 磁共振仪, $\mathrm{CDCl}_{3}$ 或 DMSO- $d_{6}$ 为溶剂, TMS 为内标. 所 用试剂均为市售分析纯或化学纯.

\section{2 合成}

3.2.14,5-二氢-3-烷氧基-4-烷基-5-氧代- $1 H-1,2,4$-三 唑甲酸苯酯(3)的合成

中间体 2 参考文献[22]合成，不经分离纯化，直接 用于下一步反应.

在 $250 \mathrm{~mL}$ 圆底烧瓶中加入 $100 \mathrm{~mL}$ 乙腈、 $60 \mathrm{mmol}$ 中间体 2 和 $70 \mathrm{mmol}$ 三乙胺, 搅拌溶解. 在冰浴冷却下, 向其中滴加 $66 \mathrm{mmol}(10.33 \mathrm{~g})$ 氯甲酸苯酯, $25 \mathrm{~min}$ 左右 滴加完毕. 于室温搅拌 $2 \mathrm{~h}$ 后, 减压脱溶, 残余物用 200 $\mathrm{mL}$ 水洗涤，有固体产生，抽滤，干燥后得到中间体 $\mathbf{3}$.

4,5-二氢-3-甲氧基-4-甲基-5-氧代- $1 H$-1,2,4-三唑甲 酸苯酯(3ae): 白色固体, 产率 74\%. m.p. 150 $151{ }^{\circ} \mathrm{C}$; ${ }^{1} \mathrm{H}$ NMR (400 MHz, DMSO- $d_{6}$ ) $\delta: 3.060$ (s, 3H, $\mathrm{NCH}_{3}$ ), $4.022\left(\mathrm{~s}, 3 \mathrm{H}\right.$, triazolone- $\left.\mathrm{OCH}_{3}\right), 7.261 \sim 7.499(\mathrm{~m}, 5 \mathrm{H}$, ArH).

4,5-二氢-3-乙氧基-4-甲基-5-氧代- $1 H$-1,2,4-三唑甲 酸苯酯(3be): 白色固体，产率 $69 \%$. m.p. $110 \sim 112{ }^{\circ} \mathrm{C}$; ${ }^{1} \mathrm{H}$ NMR (400 MHz, DMSO- $\left.d_{6}\right) \delta: 1.385(\mathrm{t}, J=6.8 \mathrm{~Hz}, 3 \mathrm{H}$, triazolone- $\left.\mathrm{OCH}_{2} \mathrm{CH}_{3}\right), 3.056\left(\mathrm{~s}, 3 \mathrm{H}, \mathrm{NCH}_{3}\right), 4.405$ (q, $J=$ $6.8 \mathrm{~Hz}, 2 \mathrm{H}$, triazolone- $\left.\mathrm{OCH}_{2} \mathrm{CH}_{3}\right), 7.255 \sim 7.503(\mathrm{~m}, 5 \mathrm{H}$, ArH). 
4,5-二氢-3-正丙氧基-4-甲基-5-氧代- $1 H-1,2,4$-三唑 甲酸苯酯(3ce): 白色固体, 产率 70\%. m.p. 92 93 ${ }^{\circ} \mathrm{C}$; ${ }^{1} \mathrm{H}$ NMR (400 MHz, DMSO- $d_{6}$ ) $\delta: 0.976$ (t, $J=7.2 \mathrm{~Hz}, 3 \mathrm{H}$, $\left.\mathrm{CH}_{2} \mathrm{CH}_{3}\right), 1.735 \sim 1.822\left(\mathrm{~m}, 2 \mathrm{H}, \mathrm{CH}_{2} \mathrm{CH}_{3}\right), 3.065(\mathrm{~s}, 3 \mathrm{H}$, triazolone- $\left.\mathrm{NCH}_{3}\right), 4.309(\mathrm{t}, J=6.4 \mathrm{~Hz}, 2 \mathrm{H}$, triazolone$\left.\mathrm{OCH}_{2}\right), 7.257 \sim 7.496(\mathrm{~m}, 5 \mathrm{H}, \mathrm{ArH})$.

4,5-二氢-3-异丙氧基-4-甲基-5-氧代- $1 H-1,2,4$-三唑 甲酸苯酯(3de): 白色固体, 产率 $65 \%$. m.p. $80 \sim 81{ }^{\circ} \mathrm{C}$; ${ }^{1} \mathrm{H}$ NMR (400 MHz, DMSO- $\left.d_{6}\right) \delta: 1.390(\mathrm{~d}, J=6.0 \mathrm{~Hz}$, $6 \mathrm{H}$, isopropyl- $\left.\mathrm{CH}_{3}\right), 3.036\left(\mathrm{~s}, 3 \mathrm{H}, \mathrm{NCH}_{3}\right), 5.035 \sim 5.096$ $(\mathrm{m}, 1 \mathrm{H}$, isopropyl-CH), $7.259 \sim 7.496(\mathrm{~m}, 5 \mathrm{H}, \mathrm{ArH})$.

4,5-二氢-3-甲氧基-4-乙基-5-氧代- $1 H-1,2,4$-三唑甲 酸苯酯(3af): 白色固体, 产率 $62 \%$. m.p. $51 \sim 53{ }^{\circ} \mathrm{C} ;{ }^{1} \mathrm{H}$ NMR (400 MHz, DMSO- $\left.d_{6}\right) \delta: 1.301$ (t, $J=7.2 \mathrm{~Hz}, 3 \mathrm{H}$, $\mathrm{NCH}_{2} \mathrm{CH}_{3}$ ), 3.684 (q, $J=7.2 \mathrm{~Hz}, 2 \mathrm{H}, \mathrm{NCH}_{2} \mathrm{CH}_{3}$ ), 4.116 (s, $3 \mathrm{H}$, triazolone- $\left.\mathrm{OCH}_{3}\right), 7.261 \sim 7.443(\mathrm{~m}, 5 \mathrm{H}, \mathrm{ArH})$.

4,5-二氢-3-乙氧基-4-乙基-5-氧代- $1 H-1,2,4$-三唑甲 酸苯酯(3bf): 白色固体, 产率 55\%. m.p. 27 $29{ }^{\circ} \mathrm{C} ;{ }^{1} \mathrm{H}$ NMR (400 MHz, DMSO- $\left.d_{6}\right) \delta: 1.193(\mathrm{t}, J=7.2 \mathrm{~Hz}, 3 \mathrm{H}$, $\left.\mathrm{NCH}_{2} \mathrm{CH}_{3}\right), 1.385$ (t, $J=6.8 \mathrm{~Hz}, 3 \mathrm{H}$, triazolone- $\mathrm{OCH}_{2}-$ $\left.\mathrm{CH}_{3}\right), 3.559$ (q, $\left.J=7.2 \mathrm{~Hz}, 2 \mathrm{H}, \mathrm{NCH}_{2} \mathrm{CH}_{3}\right), 4.414$ (q, $J=$ $7.2 \mathrm{~Hz}, 2 \mathrm{H}$, triazolone- $\left.\mathrm{OCH}_{2} \mathrm{CH}_{3}\right), 7.270 \sim 7.506(\mathrm{~m}, 5 \mathrm{H}$, $\mathrm{ArH})$.

4,5-二氢-3-正丙氧基-4-乙基-5-氧代- $1 H-1,2,4$-三唑 甲酸苯酯(3cf): 白色固体, 产率 $60 \%$. m.p. $22 \sim 23{ }^{\circ} \mathrm{C}$; ${ }^{1} \mathrm{H}$ NMR $\left(400 \mathrm{MHz}, \mathrm{CDCl}_{3}\right) \delta$ : $1.051(\mathrm{t}, J=7.2 \mathrm{~Hz}, 3 \mathrm{H}$, $\left.\mathrm{CH}_{2} \mathrm{CH}_{3}\right), 1.322\left(\mathrm{t}, J=7.2 \mathrm{~Hz}, 3 \mathrm{H}, \mathrm{NCH}_{2} \mathrm{CH}_{3}\right), 1.814 \sim$ $1.911\left(\mathrm{~m}, 2 \mathrm{H}, \mathrm{CH}_{2} \mathrm{CH}_{3}\right), 3.707$ (q, $J=7.2,14.4 \mathrm{~Hz}, 2 \mathrm{H}$, $\left.\mathrm{NCH}_{2} \mathrm{CH}_{3}\right), 4.418\left(\mathrm{t}, J=6.4 \mathrm{~Hz}, 2 \mathrm{H}\right.$, triazolone- $\mathrm{OCH}_{2}$ ), $7.263 \sim 7.454(\mathrm{~m}, 5 \mathrm{H}, \mathrm{ArH})$.

\subsection{2目标化合物 5 的合成}

$15 \mathrm{~mL}$ 乙腈中加入 $2 \mathrm{mmol}$ 磺胺 4 和 $2 \mathrm{mmol}$ 三唑甲 酸苯酯 3 , 搅拌均匀后滴加 $1.5 \mathrm{mmol} 1,8$-二氮杂二环 [5.4.0]-7-十一烯(DBU)的 $2 \mathrm{~mL}$ 乙腈溶液, 室温摚拌过 夜. 将反应液减压脱溶, 向残余物中加入 $20 \mathrm{~mL}$ 质量分 数为 $5 \%$ 的盐酸溶液, 充分摚拌. 用 $60 \mathrm{~mL}$ 二氯甲烷分 3 次对水相进行萃取, 有机相干燥, 减压脱溶, 残余物经 柱层析分离 $[V$ (石油醚) $: V$ (丙酮 $)=8 ： 1]$ 得到目标化合 物 5 .

4,5-二氢-3-甲氧基-4-甲基-5-氧代- $N$-(1-甲基-3-氯4-甲氧基甲酰基-5-吡唑磺酰基)-1H-1,2,4-三唑-1-甲酰胺 (5a): 白色固体, 产率 $72 \%$. m.p. $152 \sim 153{ }^{\circ} \mathrm{C}$; ${ }^{1} \mathrm{H}$ NMR (400 MHz, DMSO- $\left.d_{6}\right) \delta: 3.008\left(\mathrm{~s}, 3 \mathrm{H}\right.$, triazolone- $\mathrm{NCH}_{3}$ ), $3.772\left(\mathrm{~s}, 3 \mathrm{H}\right.$, pyrazole- $\left.\mathrm{CH}_{3}\right), 3.968(\mathrm{~s}, 3 \mathrm{H}$, triazolone$\left.\mathrm{OCH}_{3}\right), 4.114\left(\mathrm{~s}, 3 \mathrm{H}, \mathrm{COOCH}_{3}\right)$; ESI-FTICR-MS calcd for $\mathrm{C}_{11} \mathrm{H}_{12} \mathrm{ClN}_{6} \mathrm{O}_{7} \mathrm{~S}[\mathrm{M}-\mathrm{H}]^{-}$407.0180, found, 407.0182.

4,5-二氢-3-甲氧基-4-甲基-5-氧代- $N$-[3-(2,2,2-三氟 乙氧基)-2-吡啶磺酰基]- $1 H$-1,2,4-三唑-1-甲酰胺(5b): 白 色固体, 产率 64\%. m.p. 132 133 ${ }^{\circ} \mathrm{C} ;{ }^{1} \mathrm{H}$ NMR (400 $\left.\mathrm{MHz}, \mathrm{CDCl}_{3}\right) \delta: 3.219\left(\mathrm{~s}, 3 \mathrm{H}, \mathrm{NCH}_{3}\right), 4.093$ (s, 3H, triazolone- $\mathrm{OCH}_{3}$ ), 4.573 (q, $J=8.0 \mathrm{~Hz}, 2 \mathrm{H}, \mathrm{OCH}_{2} \mathrm{CF}_{3}$ ), $7.500 \sim 7.572(\mathrm{~m}, 2 \mathrm{H}, \mathrm{Py}-\mathrm{H}), 8.385(\mathrm{~d}, J=3.6 \mathrm{~Hz}, 1 \mathrm{H}$, Py-H), 10.723 (br, 1H, SO $2 \mathrm{NH})$. ESI-FTICR-MS calcd for $\mathrm{C}_{12} \mathrm{H}_{11} \mathrm{~F}_{3} \mathrm{~N}_{5} \mathrm{O}_{6} \mathrm{~S}[\mathrm{M}-\mathrm{H}]^{-} 410.0388$, found 410.0382 .

4,5-二氢-3-甲氧基-4-甲基-5-氧代- $N$-[2-(乙基磺酰) 咪唑并[1,2- $a$ ]吡啶-3-磺酰基]- $1 H-1,2,4$-三唑-1-甲酰胺 (5c): 白色固体，产率 75\%. m.p. 198 $199{ }^{\circ} \mathrm{C} ;{ }^{1} \mathrm{H} \mathrm{NMR}$ (400 MHz, DMSO- $\left.d_{6}\right) \delta: 1.150$ (t, $J=7.2 \mathrm{~Hz}, 3 \mathrm{H}$, $\mathrm{SO}_{2} \mathrm{CH}_{2} \mathrm{CH}_{3}$ ), 2.970 (s, 3H, $\mathrm{NCH}_{3}$ ), 3.684 (q, $J=7.2 \mathrm{~Hz}$, $\left.2 \mathrm{H}, \mathrm{SO}_{2} \mathrm{CH}_{2} \mathrm{CH}_{3}\right), 3.920\left(\mathrm{~s}, 3 \mathrm{H}\right.$, triazolone- $\left.\mathrm{OCH}_{3}\right), 7.350$ (t, $J=6.8 \mathrm{~Hz}, 1 \mathrm{H}$, Py-H), $7.670(\mathrm{t}, J=6.0 \mathrm{~Hz}, 1 \mathrm{H}$, Py-H), 7.890 (d, $J=8.8$ Hz, 1H, Py-H), 9.091 (d, $J=7.2$ $\mathrm{Hz}, \quad 1 \mathrm{H}$, Py-H). ESI-FTICR-MS calcd 443.0449 for $\mathrm{C}_{14} \mathrm{H}_{15} \mathrm{~N}_{6} \mathrm{O}_{7} \mathrm{~S}_{2}[\mathrm{M}-\mathrm{H}]^{-}$443.0449, found 443.0447.

4,5-二氢-3-甲氧基-4-甲基-5-氧代- $N$-(3-二甲基氨基 甲酰基-2-吡啶磺酰基)-1H-1,2,4-三唑-1-甲酰胺(5d): 白 色固体，产率 62\%. m.p. $165 \sim 166{ }^{\circ} \mathrm{C} ;{ }^{1} \mathrm{H}$ NMR (400 MHz, DMSO- $\left.d_{6}\right) \delta: 2.835\left(\mathrm{~s}, 3 \mathrm{H}, \mathrm{CONCH}_{3}\right), 2.995$ (s, 3H, $\left.\mathrm{CONCH}_{3}\right), 3.053\left(\mathrm{~s}, 3 \mathrm{H}, \mathrm{NCH}_{3}\right), 4.005$ (s, 3H, triazolone$\left.\mathrm{OCH}_{3}\right), 7.791 \sim 7.818(\mathrm{~m}, 1 \mathrm{H}, \mathrm{Py}-\mathrm{H}), 8.043 \sim 8.063(\mathrm{~m}$, $1 \mathrm{H}, \mathrm{Py}-\mathrm{H}), 8.740 \sim 8.754(\mathrm{~m}, 1 \mathrm{H}, \mathrm{Py}-\mathrm{H})$. ESI-FTICR-MS calcd for $\mathrm{C}_{13} \mathrm{H}_{15} \mathrm{~N}_{6} \mathrm{O}_{6} \mathrm{~S}[\mathrm{M}-\mathrm{H}]^{-}$383.0779, found 383.0780

4,5-二氢-3-甲氧基-4-甲基-5-氧代- $N$-(3-乙基磺酰 基-2-吡啶磺酰基)- $1 H$-1,2,4-三唑-1-甲酰胺(5e)：白色固 体, 产率 68\%. m.p. $164 \sim 165{ }^{\circ} \mathrm{C} ;{ }^{1} \mathrm{H}$ NMR (400 MHz, DMSO- $\left.d_{6}\right) \delta: 1.204\left(\mathrm{t}, J=7.6 \mathrm{~Hz}, 3 \mathrm{H}, \mathrm{SO}_{2} \mathrm{CH}_{2} \mathrm{CH}_{3}\right), 3.050$ (s, 3H, $\left.\mathrm{NCH}_{3}\right), 3.741$ (q, J=7.2 Hz, $2 \mathrm{H}, \mathrm{SO}_{2} \mathrm{CH}_{2} \mathrm{CH}_{3}$ ), 4.020 (s, 3H, triazolone- $\left.\mathrm{OCH}_{3}\right), 8.026(\mathrm{dd}, J=4.8,8.0 \mathrm{~Hz}$, 1H, Py-H), 8.615 (dd, $J=1.2,6.8$ Hz, 1H, Py-H), 8.979 (dd, $J=1.2,4.8 \mathrm{~Hz}, 1 \mathrm{H}$, Py-H). ESI-FTICR-MS calcd for $\mathrm{C}_{12} \mathrm{H}_{14} \mathrm{~N}_{5} \mathrm{O}_{7} \mathrm{~S}_{2}[\mathrm{M}-\mathrm{H}]^{-} \quad 404.0340$, found 404.0345.

4,5-二氢-3-甲氧基-4-甲基-5-氧代- $N$-[2-(2-氯乙氧 基)苯基磺酰基]-1H-1,2,4-三唑-1-甲酰胺(5f)：白色固体, 产率 62\%. m.p. $154 \sim 155{ }^{\circ} \mathrm{C} ;{ }^{1} \mathrm{H}$ NMR (400 MHz, DMSO- $\left.d_{6}\right) \delta: 3.052\left(\mathrm{~s}, 3 \mathrm{H}, \mathrm{NCH}_{3}\right), 3.953 \sim 3.981(\mathrm{~m}, 5 \mathrm{H}$, $\mathrm{CH}_{2} \mathrm{Cl}$, triazolone- $\left.\mathrm{OCH}_{3}\right), 4.420$ (t, $J=4.8 \mathrm{~Hz}, 2 \mathrm{H}$, Ar- $\left.\mathrm{OCH}_{2}\right), 7.190$ (t, $\left.J=7.6 \mathrm{~Hz}, 1 \mathrm{H}, \mathrm{ArH}\right), 7.314$ (d, $J=8.4$ $\mathrm{Hz}, 1 \mathrm{H}, \mathrm{ArH}), 7.707$ (t, J=7.6 Hz, 1H, ArH), 7.923 (d, $J=7.6 \mathrm{~Hz}, 1 \mathrm{H}, \mathrm{ArH}), 10.890$ (br, $\left.1 \mathrm{H}, \mathrm{SO}_{2} \mathrm{NH}\right)$. ESI- 
FTICR-MS calcd for $\mathrm{C}_{13} \mathrm{H}_{14} \mathrm{ClN}_{4} \mathrm{O}_{6} \mathrm{~S}[\mathrm{M}-\mathrm{H}]^{-}$389.0328, found 389.0326 .

4,5-二氢-3-甲氧基-4-甲基-5-氧代- $N$-[2-(2-甲氧基乙 氧基)苯基磺酰基]- $1 H-1,2,4$-三唑-1-甲酰胺 (5g): 白色固 体, 产率 71\%. m.p. $128 \sim 129{ }^{\circ} \mathrm{C} ;{ }^{1} \mathrm{H}$ NMR $(400 \mathrm{MHz}$, DMSO- $\left.d_{6}\right) \delta: 3.073\left(\mathrm{~s}, 3 \mathrm{H}, \mathrm{NCH}_{3}\right), 3.255(\mathrm{~s}, 3 \mathrm{H}$, $\mathrm{CH}_{2} \mathrm{OCH}_{3}$ ), 3.682 (t, $J=4.8 \mathrm{~Hz}, 2 \mathrm{H}, \mathrm{CH}_{2} \mathrm{OCH}_{3}$ ), 3.977 (s, $3 \mathrm{H}$, triazolone- $\left.\mathrm{OCH}_{3}\right), 4.260$ (t, $\left.J=4.8 \mathrm{~Hz}, 2 \mathrm{H}, \mathrm{Ar}-\mathrm{OCH}_{2}\right)$, 7.167 (t, $J=7.6 \mathrm{~Hz}, 1 \mathrm{H}, \mathrm{ArH}), 7.307$ (d, $J=8.4 \mathrm{~Hz}, 1 \mathrm{H}$, ArH), 7.704 (t, $J=6.8 \mathrm{~Hz}, 1 \mathrm{H}, \operatorname{ArH}), 7.908(\mathrm{~d}, J=7.2 \mathrm{~Hz}$, $1 \mathrm{H}, \mathrm{ArH}), 10.865$ (br, 1H, $\mathrm{SO}_{2} \mathrm{NH}$ ). ESI-FTICR-MS calcd for $\mathrm{C}_{14} \mathrm{H}_{17} \mathrm{~N}_{4} \mathrm{O}_{7} \mathrm{~S}[\mathrm{M}-\mathrm{H}]^{-}$385.0823, found 385.0820.

4,5-二氢-3-乙氧基-4-甲基-5-氧代- $N$ - $[2$-(2-氯乙氧 基)苯基磺酰基]-1H-1,2,4-三唑-1-甲酰胺(5h)：白色固体, 产率 60\%. m.p. $137 \sim 138{ }^{\circ} \mathrm{C}$; ${ }^{1} \mathrm{H}$ NMR $(400 \mathrm{MHz}$, DMSO- $\left.d_{6}\right) \delta: 1.346\left(\mathrm{t}, J=7.2 \mathrm{~Hz}, 3 \mathrm{H}\right.$, triazolone- $\mathrm{OCH}_{2}-$ $\mathrm{CH}_{3}$ ), $3.052\left(\mathrm{~s}, 3 \mathrm{H}, \mathrm{NCH}_{3}\right), 3.973(\mathrm{t}, J=4.8 \mathrm{~Hz}, 2 \mathrm{H}$, $\mathrm{CH}_{2} \mathrm{Cl}$ ), 4.358 (q, $J=7.2 \mathrm{~Hz}, 2 \mathrm{H}$, triazolone- $\mathrm{OCH}_{2} \mathrm{CH}_{3}$ ), 4.432 (t, $\left.J=4.8 \mathrm{~Hz}, 2 \mathrm{H}, \mathrm{Ar}-\mathrm{OCH}_{2}\right), 7.199$ (t, $J=7.6 \mathrm{~Hz}$, 1H, ArH), 7.323 (d, $J=8.4 \mathrm{~Hz}, 1 \mathrm{H}, \mathrm{ArH}), 7.722$ (t, $J=7.6$ $\mathrm{Hz}, 1 \mathrm{H}, \mathrm{ArH}$ ), 7.930 (d, J=8.0 Hz, 1H, ArH), 10.907 (br, $1 \mathrm{H}, \mathrm{SO}_{2} \mathrm{NH}$ ). MALDI-FTICR-MS calcd for $\mathrm{C}_{14} \mathrm{H}_{17} \mathrm{Cl}-$ $\mathrm{N}_{4} \mathrm{O}_{6} \mathrm{SNa}[\mathrm{M}+\mathrm{Na}]^{+} 427.0450$, found 427.0457 .

4,5-二氢-3-乙氧基-4-甲基-5-氧代- $N$-[2-(2-甲氧基乙 氧基)苯基磺酰基]-1H-1,2,4-三唑-1-甲酰胺(5i): 白色固 体, 产率 56\%. m.p. 131 132 ${ }^{\circ} \mathrm{C}$; ${ }^{1} \mathrm{H}$ NMR $(400 \mathrm{MHz}$, DMSO- $\left.d_{6}\right) \delta: 1.347$ (t, $J=6.8 \mathrm{~Hz}, 3 \mathrm{H}$, triazolone- $\mathrm{OCH}_{2}-$ $\left.\mathrm{CH}_{3}\right), 3.071\left(\mathrm{~s}, 3 \mathrm{H}, \mathrm{NCH}_{3}\right), 3.254\left(\mathrm{~s}, 3 \mathrm{H}, \mathrm{CH}_{2} \mathrm{OCH}_{3}\right)$, 3.679 (t, $\left.J=4.8 \mathrm{~Hz}, 2 \mathrm{H}, \mathrm{CH}_{2} \mathrm{OCH}_{3}\right), 4.239$ (t, $J=5.2 \mathrm{~Hz}$, $2 \mathrm{H}, \mathrm{Ar}-\mathrm{OCH}_{2}$ ), 4.359 (q, $J=6.8 \mathrm{~Hz}, 2 \mathrm{H}$, triazolone$\left.\mathrm{OCH}_{2} \mathrm{CH}_{3}\right), 7.170$ (t, $\left.J=7.6 \mathrm{~Hz}, 1 \mathrm{H}, \mathrm{ArH}\right), 7.310$ (d, $J=$ $8.4 \mathrm{~Hz}, 1 \mathrm{H}, \mathrm{ArH}), 7.707$ (t, $J=7.6 \mathrm{~Hz}, 1 \mathrm{H}, \mathrm{ArH}), 7.911$ (d, $J=8.0 \mathrm{~Hz}, 1 \mathrm{H}, \mathrm{ArH}), 10.889$ (br, $\left.1 \mathrm{H}, \mathrm{SO}_{2} \mathrm{NH}\right)$. MALDIFTICR-MS calcd for $\mathrm{C}_{15} \mathrm{H}_{20} \mathrm{~N}_{4} \mathrm{O}_{7} \mathrm{SNa} \quad\left[\mathrm{M}+\mathrm{Na}{ }^{+}\right.$ 423.0945, found 423.0948.

4,5-二氢-3-正丙氧基-4-甲基-5-氧代- $N$-[2-(2-氯乙氧 基)苯基磺酰基]-1 $H$-1,2,4-三唑-1-甲酰胺 (5j): 白色固体, 产率 68\%. m.p. $136 \sim 138{ }^{\circ} \mathrm{C} ;{ }^{1} \mathrm{H}$ NMR $(400 \mathrm{MHz}$, DMSO- $\left.d_{6}\right) \delta: 0.941\left(\mathrm{t}, J=7.2 \mathrm{~Hz}, 3 \mathrm{H}, \mathrm{CH}_{2} \mathrm{CH}_{3}\right), 1.698 \sim$ $1.787\left(\mathrm{~m}, 2 \mathrm{H}, \mathrm{CH}_{2} \mathrm{CH}_{3}\right), 3.060\left(\mathrm{~s}, 3 \mathrm{H}, \mathrm{NCH}_{3}\right), 3.974(\mathrm{t}$, $\left.J=4.8 \mathrm{~Hz}, 2 \mathrm{H}, \mathrm{CH}_{2} \mathrm{Cl}\right), 4.265$ (t, $J=6.4 \mathrm{~Hz}, 2 \mathrm{H}$, triazolone- $\mathrm{OCH}_{2}$ ), 4.431 (t, $J=4.8 \mathrm{~Hz}, 2 \mathrm{H}, \mathrm{Ar}-\mathrm{OCH}_{2}$ ), 7.198 (t, $J=7.6 \mathrm{~Hz}, 1 \mathrm{H}, \mathrm{ArH}), 7.323$ (d, $J=8.0 \mathrm{~Hz}, 1 \mathrm{H}, \mathrm{ArH})$, 7.720 (t, $J=7.6 \mathrm{~Hz}, 1 \mathrm{H}, \mathrm{ArH}), 7.929$ (d, $J=7.6 \mathrm{~Hz}, 1 \mathrm{H}$, $\mathrm{ArH}), 10.894$ (br, $1 \mathrm{H}, \mathrm{SO}_{2} \mathrm{NH}$ ). MALDI-FTICR-MS calcd for $\mathrm{C}_{15} \mathrm{H}_{19} \mathrm{ClN}_{4} \mathrm{O}_{6} \mathrm{SNa}[\mathrm{M}+\mathrm{Na}]^{+}$441.0606, found 441.0610 .

4,5-二氢-3-正丙氧基-4-甲基-5-氧代- $N$-[2-(2-甲氧基 乙氧基)苯基磺酰基]-1 $H$-1,2,4-三唑-1-甲酰胺(5k): 白色 固体, 产率 59\%. m.p. 122 123 ${ }^{\circ} \mathrm{C} ;{ }^{1} \mathrm{H}$ NMR $(400 \mathrm{MHz}$, DMSO- $\left.d_{6}\right) \delta: 0.941\left(\mathrm{t}, J=7.2 \mathrm{~Hz}, 3 \mathrm{H}, \mathrm{CH}_{2} \mathrm{CH}_{3}\right), 1.699 \sim$ $1.786\left(\mathrm{~m}, 2 \mathrm{H}, \mathrm{CH}_{2} \mathrm{CH}_{3}\right), 3.078\left(\mathrm{~s}, 3 \mathrm{H}, \mathrm{NCH}_{3}\right), 3.254$ (s, $3 \mathrm{H}, \mathrm{CH}_{2} \mathrm{OCH}_{3}$ ), 3.682 (t, $J=4.4 \mathrm{~Hz}, 2 \mathrm{H}, \mathrm{CH}_{2} \mathrm{OCH}_{3}$ ), $4.250 \sim 4.281\left(\mathrm{~m}, 4 \mathrm{H}\right.$, triazolone- $\left.\mathrm{OCH}_{2}, \mathrm{Ar}-\mathrm{OCH}_{2}\right), 7.169$ (t, $J=7.6 \mathrm{~Hz}, 1 \mathrm{H}, \mathrm{ArH}), 7.307$ (d, $J=8.4 \mathrm{~Hz}, 1 \mathrm{H}, \mathrm{ArH})$, 7.704 (t, $J=7.6 \mathrm{~Hz}, 1 \mathrm{H}, \mathrm{ArH}), 7.908(\mathrm{~d}, J=7.2 \mathrm{~Hz}, 1 \mathrm{H}$, ArH), 10.865 (br, 1H, $\mathrm{SO}_{2} \mathrm{NH}$ ). MALDI-FTICR-MS calcd for $\mathrm{C}_{16} \mathrm{H}_{22} \mathrm{~N}_{4} \mathrm{O}_{7} \mathrm{SNa}[\mathrm{M}+\mathrm{Na}]^{+} 437.1101$, found 437.1100 .

4,5-二氢-3-正丙氧基-4-甲基-5-氧代- $N$-(1-甲基-3氯-4-甲氧基甲酰基-5-吡唑磺酰基)-1H-1,2,4-三唑-1-甲 酰胺(5I): 白色固体, 产率 48\%. m.p. $135 \sim 136{ }^{\circ} \mathrm{C} ;{ }^{1} \mathrm{H}$ NMR $\left(400 \mathrm{MHz}, \mathrm{CDCl}_{3}\right) \delta: 1.006(\mathrm{t}, J=7.2 \mathrm{~Hz}, 3 \mathrm{H}$, $\mathrm{CH}_{2} \mathrm{CH}_{3}$ ), $1.791 \sim 1.880\left(\mathrm{~m}, 2 \mathrm{H}, \mathrm{CH}_{2} \mathrm{CH}_{3}\right), 3.219$ (s, $3 \mathrm{H}$, triazolone- $\mathrm{NCH}_{3}$ ), 3.924 (s, $3 \mathrm{H}$, pyrazole- $\left.\mathrm{CH}_{3}\right), 4.311$ (s, $3 \mathrm{H}, \mathrm{COOCH}_{3}$ ), 4.376 (t, $J=6.8 \mathrm{~Hz}, 2 \mathrm{H}$, triazolone- $\mathrm{OCH}_{2}$ ), $11.097\left(\mathrm{br}, 1 \mathrm{H}, \mathrm{SO}_{2} \mathrm{NH}\right)$. MALDI-FTICR-MS calcd for $\mathrm{C}_{13} \mathrm{H}_{17} \mathrm{ClN}_{6} \mathrm{O}_{7} \mathrm{SNa}[\mathrm{M}+\mathrm{Na}]^{+}$459.0460, found 459.0462.

4,5-二氢-3-正丙氧基-4-甲基-5-氧代- $N$-[3-(2,2,2-三 氟乙氧基)-2-吡啶磺酰基]- $1 H-1,2,4$-三唑-1-甲酰胺 (5m): 白色固体, 产率 50\%. m.p. 138 139 ${ }^{\circ} \mathrm{C} ;{ }^{1} \mathrm{H}$ NMR (400 $\left.\mathrm{MHz}, \mathrm{DMSO}-d_{6}\right) \delta: 0.967\left(\mathrm{t}, J=7.2 \mathrm{~Hz}, 3 \mathrm{H}, \mathrm{CH}_{2} \mathrm{CH}_{3}\right.$ ), $1.728 \sim 1.816\left(\mathrm{~m}, 2 \mathrm{H}, \mathrm{CH}_{2} \mathrm{CH}_{3}\right), 3.087\left(\mathrm{~s}, 3 \mathrm{H}, \mathrm{NCH}_{3}\right)$, $4.312\left(\mathrm{t}, J=6.8 \mathrm{~Hz}, 2 \mathrm{H}\right.$, triazolone- $\left.\mathrm{OCH}_{2}\right), 5.067$ (q, $J=$ $\left.8.4 \mathrm{~Hz}, 2 \mathrm{H}, \mathrm{OCH}_{2} \mathrm{CF}_{3}\right), 7.794 \sim 7.826(\mathrm{~m}, 1 \mathrm{H}, \mathrm{Py}-\mathrm{H})$, 7.983 (d, $J=8.8 \mathrm{~Hz}, 1 \mathrm{H}, \mathrm{Py}-\mathrm{H}), 8.338$ (d, $J=4.4 \mathrm{~Hz}, 1 \mathrm{H}$, Py-H). MALDI-FTICR-MS calcd for $\mathrm{C}_{14} \mathrm{H}_{16} \mathrm{~F}_{3} \mathrm{~N}_{5} \mathrm{O}_{6} \mathrm{SNa}$ $[\mathrm{M}+\mathrm{Na}]^{+}$462.0666, found 462.0665 .

4,5-二氢-3-正丙氧基-4-甲基-5-氧代- $N$-[2-(乙基磺 酰)咪唑并[1,2- $a$ ]吡啶-3-磺酰基]-1H-1,2,4-三唑-1-甲酰 胺 $(5 n)$ : 白色固体, 产率 58\%. m.p. $168 \sim 169{ }^{\circ} \mathrm{C} ;{ }^{1} \mathrm{H}$ NMR $\left(400 \mathrm{MHz}, \mathrm{CDCl}_{3}\right) \delta: 0.973(\mathrm{t}, J=7.2 \mathrm{~Hz}, 3 \mathrm{H}$, $\left.\mathrm{CH}_{2} \mathrm{CH}_{3}\right), 1.430$ (t, $\left.J=7.6 \mathrm{~Hz}, 3 \mathrm{H}, \mathrm{SO}_{2} \mathrm{CH}_{2} \mathrm{CH}_{3}\right), 1.750 \sim$ $1.838\left(\mathrm{~m}, 2 \mathrm{H}, \mathrm{CH}_{2} \mathrm{CH}_{3}\right), 3.181\left(\mathrm{~s}, 3 \mathrm{H}, \mathrm{NCH}_{3}\right), 3.588$ (q, $\left.J=7.6 \mathrm{~Hz}, 2 \mathrm{H}, \mathrm{SO}_{2} \mathrm{CH}_{2} \mathrm{CH}_{3}\right), 4.309(\mathrm{t}, J=6.8 \mathrm{~Hz}, 2 \mathrm{H}$, triazolone- $\mathrm{OCH}_{2}$ ), $7.246(\mathrm{t}, J=6.8 \mathrm{~Hz}, 1 \mathrm{H}, \mathrm{Py}-\mathrm{H}), 7.606$ (t, $J=7.6 \mathrm{~Hz}, 1 \mathrm{H}, \mathrm{Py}-\mathrm{H}), 7.860$ (d, $J=8.8 \mathrm{~Hz}, 1 \mathrm{H}, \mathrm{Py}-\mathrm{H})$, 9.350 (d, $J=7.2 \mathrm{~Hz}, 1 \mathrm{H}, \mathrm{Py}-\mathrm{H}), 11.008$ (br, $1 \mathrm{H}, \mathrm{SO}_{2} \mathrm{NH}$ ). MALDI-FTICR-MS calcd for $\mathrm{C}_{16} \mathrm{H}_{20} \mathrm{~N}_{6} \mathrm{O}_{7} \mathrm{~S}_{2} \mathrm{Na}[\mathrm{M}+\mathrm{Na}]^{+}$ 495.0727, found 495.0723.

4,5-二氢-3-正丙氧基-4-甲基-5-氧代- $N$-(3-乙基磺酰 
基-2-吡啶磺酰基)-1H-1,2,4-三唑-1-甲酰胺(5o): 白色固 体, 产率 53\%. m.p. 153 $154{ }^{\circ} \mathrm{C} ;{ }^{1} \mathrm{H}$ NMR (400 MHz, $\left.\mathrm{CDCl}_{3}\right) \delta: 0.997\left(\mathrm{t}, J=7.6 \mathrm{~Hz}, 3 \mathrm{H}, \mathrm{CH}_{2} \mathrm{CH}_{3}\right), 1.346(\mathrm{t}, J=$ $\left.7.2 \mathrm{~Hz}, 3 \mathrm{H}, \quad \mathrm{SO}_{2} \mathrm{CH}_{2} \mathrm{CH}_{3}\right), 1.776 \sim 1.864(\mathrm{~m}, 2 \mathrm{H}$, $\mathrm{CH}_{2} \mathrm{CH}_{3}$ ), 3.220 (s, 3H, $\mathrm{NCH}_{3}$ ), 3.760 (q, $J=7.2 \mathrm{~Hz}, 2 \mathrm{H}$, $\left.\mathrm{SO}_{2} \mathrm{CH}_{2} \mathrm{CH}_{3}\right), 4.348\left(\mathrm{t}, J=6.8 \mathrm{~Hz}, 2 \mathrm{H}\right.$, triazolone- $\left.\mathrm{OCH}_{2}\right)$, 7.753 (dd, $J=4.4,7.2 \mathrm{~Hz}, 1 \mathrm{H}, \mathrm{Py}-\mathrm{H}), 8.597$ (d, $J=8.0 \mathrm{~Hz}$, 1H, Py-H), 8.850 (d, J=3.6 Hz, 1H, Py-H), 10.865 (br, $1 \mathrm{H}, \mathrm{SO}_{2} \mathrm{NH}$ ). MALDI-FTICR-MS calcd for $\mathrm{C}_{14} \mathrm{H}_{19} \mathrm{~N}_{5} \mathrm{O}_{7^{-}}$ $\mathrm{S}_{2} \mathrm{Na}[\mathrm{M}+\mathrm{Na}]^{+}$456.0618, found 456.0617.

4,5-二氢-3-异丙氧基-4-甲基-5-氧代- $N$-[2-(2-氯乙氧 基)苯基磺酰基]- $1 H-1,2,4$-三唑-1-甲酰胺(5p): 白色固体, 产率 70\%. m.p. $149 \sim 150{ }^{\circ} \mathrm{C}$; ${ }^{1} \mathrm{H}$ NMR $(400 \mathrm{MHz}$, DMSO- $\left.d_{6}\right) \delta: 1.346\left(\mathrm{~d}, J=6.0 \mathrm{~Hz}, 6 \mathrm{H}\right.$, isopropyl- $\left.\mathrm{CH}_{3}\right)$, $3.030\left(\mathrm{~s}, 3 \mathrm{H}, \mathrm{NCH}_{3}\right), 3.971\left(\mathrm{t}, J=4.8 \mathrm{~Hz}, 2 \mathrm{H}, \mathrm{CH}_{2} \mathrm{Cl}\right)$, $4.434\left(\mathrm{t}, J=4.8 \mathrm{~Hz}, 2 \mathrm{H}, \mathrm{ArOCH}_{2}\right), 4.977 \sim 5.038(\mathrm{~m}, 1 \mathrm{H}$, isopropyl-CH), 7.198 (t, J=7.6 Hz, 1H, ArH), 7.323 (d, $J=8.0 \mathrm{~Hz}, 1 \mathrm{H}, \mathrm{ArH}), 7.719$ (t, $J=7.6 \mathrm{~Hz}, 1 \mathrm{H}, \mathrm{ArH}), 7.929$ (d, $J=7.6 \mathrm{~Hz}, 1 \mathrm{H}, \mathrm{ArH}$ ), 10.928 (br, $1 \mathrm{H}, \mathrm{SO}_{2} \mathrm{NH}$ ). MALDI-FTICR-MS calcd for $\mathrm{C}_{15} \mathrm{H}_{19} \mathrm{ClN}_{4} \mathrm{O}_{6} \mathrm{SNa}[\mathrm{M}+$ $\mathrm{Na}]^{+}$441.0606, found 441.0603.

4,5-二氢-3-异丙氧基-4-甲基-5-氧代- $N$-[2-(2-甲氧基 乙氧基)苯基磺酰基]-1H-1,2,4-三唑-1-甲酰胺(5q): 白色 固体, 产率 66\%. m.p. $190 \sim 120{ }^{\circ} \mathrm{C} ;{ }^{1} \mathrm{H}$ NMR $(400 \mathrm{MHz}$, DMSO- $\left.d_{6}\right) \delta: 1.349\left(\mathrm{~d}, J=6.0 \mathrm{~Hz}, 6 \mathrm{H}\right.$, isopropyl- $\left.\mathrm{CH}_{3}\right)$, $3.050\left(\mathrm{~s}, 3 \mathrm{H}, \mathrm{NCH}_{3}\right), 3.252\left(\mathrm{~s}, 3 \mathrm{H}, \mathrm{CH}_{2} \mathrm{OCH}_{3}\right), 3.685$ (t, $\left.J=4.4 \mathrm{~Hz}, 2 \mathrm{H}, \mathrm{CH}_{2} \mathrm{OCH}_{3}\right), 4.269$ (t, $J=4.4 \mathrm{~Hz}, 2 \mathrm{H}$, $\left.\mathrm{ArOCH}_{2}\right), 4.972 \sim 5.032(\mathrm{~m}, 1 \mathrm{H}$, isopropyl-CH), $7.174(\mathrm{t}$, $J=7.6 \mathrm{~Hz}, 1 \mathrm{H}, \mathrm{ArH}), 7.313(\mathrm{~d}, J=8.4 \mathrm{~Hz}, 1 \mathrm{H}, \mathrm{ArH})$, 7.710 (t, $J=7.6 \mathrm{~Hz}, 1 \mathrm{H}, \mathrm{ArH}), 7.902$ (d, $J=7.6 \mathrm{~Hz}, 1 \mathrm{H}$, ArH), 10.921 (br, 1H, $\mathrm{SO}_{2} \mathrm{NH}$ ). MALDI-FTICR-MS calcd for $\mathrm{C}_{16} \mathrm{H}_{22} \mathrm{~N}_{4} \mathrm{O}_{7} \mathrm{SNa}[\mathrm{M}+\mathrm{Na}]^{+}$437.1101, found 437.1103.

4,5-二氢-3-甲氧基-4-乙基-5-氧代- $N$-[2-(2-氯乙氧 基)苯基磺酰基]- $1 H-1,2,4$-三唑-1-甲酰胺(5r)：白色固体, 产率 77\%. m.p. 147 $149{ }^{\circ} \mathrm{C} ;{ }^{1} \mathrm{H} \mathrm{NMR}\left(400 \mathrm{MHz}, \mathrm{CDCl}_{3}\right)$ $\delta: 1.281\left(\mathrm{t}, J=7.2 \mathrm{~Hz}, 3 \mathrm{H}, \mathrm{NCH}_{2} \mathrm{CH}_{3}\right), 3.661$ (q, $J=7.2$ $\left.\mathrm{Hz}, 2 \mathrm{H}, \mathrm{NCH}_{2} \mathrm{CH}_{3}\right), 3.904\left(\mathrm{t}, J=5.6 \mathrm{~Hz}, 2 \mathrm{H}, \mathrm{CH}_{2} \mathrm{Cl}\right)$, $4.054\left(\mathrm{~s}, 3 \mathrm{H}\right.$, triazolone- $\left.\mathrm{OCH}_{3}\right), 4.356(\mathrm{t}, J=5.6 \mathrm{~Hz}, 2 \mathrm{H}$, Ar- $\left.\mathrm{OCH}_{2}\right), 6.984$ (d, $\left.J=8.4 \mathrm{~Hz}, 1 \mathrm{H}, \mathrm{ArH}\right), 7.126$ (t, $J=7.6$ $\mathrm{Hz}, 1 \mathrm{H}, \mathrm{ArH}), 7.569$ (t, J=7.2 Hz, 1H, ArH), 8.136 (d, $J=8.0 \mathrm{~Hz}, 1 \mathrm{H}, \mathrm{ArH}), 10.673\left(\mathrm{~s}, 1 \mathrm{H}, \mathrm{SO}_{2} \mathrm{NH}\right)$. MALDIFTICR-MS calcd for $\mathrm{C}_{14} \mathrm{H}_{17} \mathrm{ClN}_{4} \mathrm{O}_{6} \mathrm{SNa}\left[\mathrm{M}+\mathrm{Na}{ }^{+}\right.$ 427.0450, found 427.0450.

4,5-二氢-3-甲氧基-4-乙基-5-氧代- $N$-[2-(2-甲氧基乙 氧基)苯基磺酰基]- $1 H-1,2,4$-三唑-1-甲酰胺(5s)：白色固
体，产率 69\%. m.p. 107 $108{ }^{\circ} \mathrm{C} ;{ }^{1} \mathrm{H}$ NMR (400 MHz, $\left.\mathrm{CDCl}_{3}\right) \delta: 1.287\left(\mathrm{t}, J=7.2 \mathrm{~Hz}, 3 \mathrm{H}, \mathrm{NCH}_{2} \mathrm{CH}_{3}\right), 3.414$ (s, $3 \mathrm{H}, \mathrm{CH}_{2} \mathrm{OCH}_{3}$ ), 3.664 (q, $J=7.2 \mathrm{~Hz}, 2 \mathrm{H}, \mathrm{NCH}_{2} \mathrm{CH}_{3}$ ), $3.806\left(\mathrm{t}, J=4.8 \mathrm{~Hz}, 2 \mathrm{H}, \mathrm{CH}_{2} \mathrm{OCH}_{3}\right), 4.056$ (s, 3H, triazolone- $\mathrm{OCH}_{3}$ ), 4.233 (t, $\left.J=4.8 \mathrm{~Hz}, 2 \mathrm{H}, \mathrm{ArOCH}_{2}\right), 7.016$ (d, $J=8.4 \mathrm{~Hz}, 1 \mathrm{H}, \mathrm{ArH}), 7.087$ (t, $J=7.6 \mathrm{~Hz}, 1 \mathrm{H}, \mathrm{ArH})$, 7.553 (t, $J=8.0 \mathrm{~Hz}, 1 \mathrm{H}, \mathrm{ArH}), 8.108$ (d, $J=7.6 \mathrm{~Hz}, 1 \mathrm{H}$, ArH), 10.681 (s, 1H, SO $\left.\mathrm{SO}_{2} \mathrm{NH}\right)$. MALDI-FTICR-MS calcd for $\mathrm{C}_{15} \mathrm{H}_{20} \mathrm{~N}_{4} \mathrm{O}_{7} \mathrm{SNa}[\mathrm{M}+\mathrm{Na}]^{+}$423.0945, found 423.0947 .

4,5-二氢-3-乙氧基-4-乙基-5-氧代- $N$-[2-(2-氯乙氧 基)苯基磺酰基]-1 $H$-1,2,4-三唑-1-甲酰胺(5t): 白色固体, 产率 55\%. m.p. $130 \sim 131{ }^{\circ} \mathrm{C} ;{ }^{1} \mathrm{H}$ NMR $(400 \mathrm{MHz}$, DMSO- $\left.d_{6}\right) \delta: 1.175\left(\mathrm{t}, J=6.8 \mathrm{~Hz}, 3 \mathrm{H}, \mathrm{NCH}_{2} \mathrm{CH}_{3}\right), 1.349$ (t, $J=7.2 \mathrm{~Hz}, 3 \mathrm{H}$, triazolone- $\mathrm{OCH}_{2} \mathrm{CH}_{3}$ ), 3.555 (q, $J=6.4$ $\mathrm{Hz}, 2 \mathrm{H}, \mathrm{NCH}_{2} \mathrm{CH}_{3}$ ), 3.977 ( $\mathrm{t}, J=4.8 \mathrm{~Hz}, 2 \mathrm{H}, \mathrm{CH}_{2} \mathrm{Cl}$ ), 4.370 (q, $J=6.4 \mathrm{~Hz}, 2 \mathrm{H}$, triazolone- $\left.\mathrm{OCH}_{2} \mathrm{CH}_{3}\right), 4.443$ (t, $\left.J=4.8 \mathrm{~Hz}, 2 \mathrm{H}, \mathrm{ArOCH}_{2}\right), 7.200$ (t, $\left.J=7.6 \mathrm{~Hz}, 1 \mathrm{H}, \mathrm{ArH}\right)$, $7.326(\mathrm{~d}, J=8.4 \mathrm{~Hz}, 1 \mathrm{H}, \operatorname{ArH}), 7.724$ (t, $J=8.0 \mathrm{~Hz}, 1 \mathrm{H}$, ArH), 7.933 (d, $J=7.6 \mathrm{~Hz}, 1 \mathrm{H}, \mathrm{ArH}), 10.932$ (br, 1H, $\mathrm{SO}_{2} \mathrm{NH}$ ). MALDI-FTICR-MS calcd for $\mathrm{C}_{15} \mathrm{H}_{19} \mathrm{ClN}_{4} \mathrm{O}_{6} \mathrm{SNa}$ $[\mathrm{M}+\mathrm{Na}]^{+}$441.0606, found 441.0606 .

4,5-二氢-3-乙氧基-4-乙基-5-氧代- $N$-[2-(2-甲氧基乙 氧基)苯基磺酰基]- $1 H-1,2,4$-三唑-1-甲酰胺(5u): 白色固 体, 产率 59\%. m.p. 105 106 ${ }^{\circ} \mathrm{C} ;{ }^{1} \mathrm{H}$ NMR (400 MHz, DMSO- $\left.d_{6}\right) \delta: 1.192\left(\mathrm{t}, J=6.8 \mathrm{~Hz}, 3 \mathrm{H}, \mathrm{NCH}_{2} \mathrm{CH}_{3}\right), 1.350$ $\left(\mathrm{t}, J=6.8 \mathrm{~Hz}, 3 \mathrm{H}\right.$, triazolone- $\left.\mathrm{OCH}_{2} \mathrm{CH}_{3}\right), 3.251(\mathrm{~s}, 3 \mathrm{H}$, $\mathrm{CH}_{2} \mathrm{OCH}_{3}$ ), 3.572 (q, $\left.J=6.8 \mathrm{~Hz}, 2 \mathrm{H}, \mathrm{NCH}_{2} \mathrm{CH}_{3}\right), 3.685$ (t, $\left.J=4.8 \mathrm{~Hz}, 2 \mathrm{H}, \mathrm{CH}_{2} \mathrm{OCH}_{3}\right), 4.273(\mathrm{t}, J=4.8 \mathrm{~Hz}, 2 \mathrm{H}$, $\left.\mathrm{ArOCH}_{2}\right), 4.369\left(\mathrm{q}, J=6.8 \mathrm{~Hz}, 2 \mathrm{H}\right.$, triazolone- $\mathrm{OCH}_{2}-$ $\left.\mathrm{CH}_{3}\right), 7.172$ (t, $\left.J=7.6 \mathrm{~Hz}, 1 \mathrm{H}, \mathrm{ArH}\right), 7.314$ (d, $J=8.4 \mathrm{~Hz}$, $1 \mathrm{H}, \mathrm{ArH}), 7.710$ (t, $J=7.6 \mathrm{~Hz}, 1 \mathrm{H}, \mathrm{ArH}), 7.913$ (d, $J=7.6$ $\mathrm{Hz}, 1 \mathrm{H}, \mathrm{ArH}$ ), 10.910 (br, 1H, $\mathrm{SO}_{2} \mathrm{NH}$ ). MALDI-FTICRMS calcd for $\mathrm{C}_{16} \mathrm{H}_{22} \mathrm{~N}_{4} \mathrm{O}_{7} \mathrm{SNa}[\mathrm{M}+\mathrm{Na}]^{+} 437.1101$, found 437.1100.

4,5-二氢-3-正丙氧基-4-乙基-5-氧代- $N$-[2-(2-氯乙氧 基)苯基磺酰基]- $1 H$-1,2,4-三唑-1-甲酰胺(5v)：白色固体， 产率 64\%. m.p. 136 $137{ }^{\circ} \mathrm{C}$; ${ }^{1} \mathrm{H}$ NMR $(400 \mathrm{MHz}$, DMSO- $\left.d_{6}\right) \delta: 0.941\left(\mathrm{t}, J=7.2 \mathrm{~Hz}, 3 \mathrm{H}, \mathrm{CH}_{2} \mathrm{CH}_{3}\right), 1.179(\mathrm{t}$, $\left.J=6.8 \mathrm{~Hz}, 3 \mathrm{H}, \mathrm{NCH}_{2} \mathrm{CH}_{3}\right), 1.703 \sim 1.788(\mathrm{~m}, 2 \mathrm{H}$, $\mathrm{CH}_{2} \mathrm{CH}_{3}$ ), 3.564 (q, $J=6.8 \mathrm{~Hz}, 2 \mathrm{H}, \mathrm{NCH}_{2} \mathrm{CH}_{3}$ ), 3.978 (t, $\left.J=4.4 \mathrm{~Hz}, 2 \mathrm{H}, \mathrm{CH}_{2} \mathrm{Cl}\right), 4.279(\mathrm{t}, J=6.4 \mathrm{~Hz}, 2 \mathrm{H}$, triazolone- $\left.\mathrm{OCH}_{2}\right), 4.442\left(\mathrm{t}, J=4.8 \mathrm{~Hz}, 2 \mathrm{H}, \mathrm{ArOCH}_{2}\right)$, 7.200 (t, $J=7.6 \mathrm{~Hz}, 1 \mathrm{H}, \mathrm{ArH}), 7.325$ (d, $J=8.4 \mathrm{~Hz}, 1 \mathrm{H}$, ArH), 7.723 (t, $J=7.6 \mathrm{~Hz}, 1 \mathrm{H}, \mathrm{ArH}), 7.933$ (d, $J=7.6 \mathrm{~Hz}$, $1 \mathrm{H}, \mathrm{ArH}$ ), 10.927 (br, 1H, $\mathrm{SO}_{2} \mathrm{NH}$ ). MALDI-FTICR-MS 
calcd for $\mathrm{C}_{16} \mathrm{H}_{21} \mathrm{ClN}_{4} \mathrm{O}_{6} \mathrm{SNa}[\mathrm{M}+\mathrm{Na}]^{+} 455.0763$, found 455.0767.

4,5-二氢-3-正丙氧基-4-乙基-5-氧代- $N$-[2-(2-甲氧基 乙氧基)苯基磺酰基]-1 $H$-1,2,4-三唑-1-甲酰胺(5w)：白色 固体, 产率 73\%. m.p. 126 127 ${ }^{\circ} \mathrm{C} ;{ }^{1} \mathrm{H}$ NMR $(400 \mathrm{MHz}$, DMSO- $\left.d_{6}\right) \delta: 0.943\left(\mathrm{t}, J=7.2 \mathrm{~Hz}, 3 \mathrm{H}, \mathrm{CH}_{2} \mathrm{CH}_{3}\right), 1.196$ (t, $\left.J=7.2 \mathrm{~Hz}, 3 \mathrm{H}, \mathrm{NCH}_{2} \mathrm{CH}_{3}\right), 1.705 \sim 1.790(\mathrm{~m}, 2 \mathrm{H}$, $\mathrm{CH}_{2} \mathrm{CH}_{3}$ ), 3.251 (s, $3 \mathrm{H}, \mathrm{CH}_{2} \mathrm{OCH}_{3}$ ), 3.581 (q, $J=7.2 \mathrm{~Hz}$, $\left.2 \mathrm{H}, \mathrm{NCH}_{2} \mathrm{CH}_{3}\right), 3.685$ (t, $J=4.4 \mathrm{~Hz}, 2 \mathrm{H}, \mathrm{CH}_{2} \mathrm{OCH}_{3}$ ), $4.262 \sim 4.293\left(\mathrm{~m}, 4 \mathrm{H}\right.$, triazolone- $\left.\mathrm{OCH}_{2}, \mathrm{ArOCH}_{2}\right), 7.172$ (t, $J=7.6 \mathrm{~Hz}, 1 \mathrm{H}, \mathrm{ArH}), 7.313$ (d, $J=8.4 \mathrm{~Hz}, 1 \mathrm{H}, \mathrm{ArH})$, 7.710 (t, $J=8.0 \mathrm{~Hz}, 1 \mathrm{H}, \mathrm{ArH}), 7.911$ (d, $J=7.6 \mathrm{~Hz}, 1 \mathrm{H}$, $\mathrm{ArH}), 10.907$ (br, $1 \mathrm{H}, \mathrm{SO}_{2} \mathrm{NH}$ ). MALDI-FTICR-MS calcd for $\mathrm{C}_{17} \mathrm{H}_{24} \mathrm{~N}_{4} \mathrm{O}_{7} \mathrm{SNa}[\mathrm{M}+\mathrm{Na}]^{+} 451.1258$, found 451.1261.

\section{3 晶体结构测定}

取约 $50 \mathrm{mg}$ 化合物 $\mathbf{5 a}$ 溶于适量丙酮中, 室温下缓慢 挥发溶剂得无色透明块状晶体. 选取大小为 $0.22 \mathrm{~mm} \times$ $0.18 \mathrm{~mm} \times 0.16 \mathrm{~mm}$ 的晶体, 在 Rigaku Saturn CCD 衍射 仪上, 于 $113(2) \mathrm{K}$ 下用 $\mathrm{Mo} \mathrm{K} \alpha$ 射线 $(\lambda=0.71075 \AA)$ 以 $\omega$ 扫描方式在 $2.00^{\circ} \leqslant \theta \leqslant 27.96^{\circ}$ 范围内共收集到 13322 个 衍射点, 其中 $I>2 \sigma(I)$ 的可观测衍射点 $3803\left(R_{\text {int }}=\right.$ 0.0956). 所有数据经过半经验方法进行吸收校正. 晶体 结构用 Bruker SHELXTL 程序包求解, 非氢原子坐标用 直接法解出, 最小二乘法对非氢原子进行各种异性温度 因子修正. 化合物 5a 属于单斜晶系, 晶胞参数为 $a=$ 9.1370(9) $\AA, b=20.384(2) \AA, c=8.7700(7) \AA, \alpha=90^{\circ}$, $\beta=103.595(6)^{\circ}, \gamma=90^{\circ}, V=1587.6(3) \AA^{3}, Z=4, D_{\mathrm{c}}=$ $1.710 \mathrm{mg} / \mathrm{m}^{3}, \mu=0.426 \mathrm{~mm}^{-1}, F(000)=840$. 晶体结构数 据存于英国剑桥数据中心, CCDC 号为 903774 .

\section{3 除草活性测试}

用油菜平皿法、稗草小杯法和盆栽法 ${ }^{[23 \sim 25]}$ 对 $1 \sim 23$ 号化合物进行了除草活性测试, 并以商品除草剂醚磺 隆, 醚苯磺隆和单嘧磺酯作为对照药剂.

盆栽法: 在直径 $8 \mathrm{~cm}$ 的塑料小杯中放入一定量的 土, 加入一定量的水, 播种后覆盖一定厚度的土壤, 于 花房中培养, 幼苗出土前以塑料布覆盖. 每天加以定量 的清水以保持正常生长. 测试试材: 油菜 (Brassica napus)、反枝苋(Amaranthus retroflexus)、稗草(Echinochloa crusgalli)和马唐(Digitaria adscendens). 施药方法 为喷施, 处理 $21 \mathrm{~d}$ 后调查结果, 测定地上部鲜重, 以鲜 重抑制百分数来表示药效.

平血法: 试验靶标为油菜(Brassica napus). 直径 6 $\mathrm{cm}$ 的培养血中铺好一张直径 $5.6 \mathrm{~cm}$ 的滤纸, 加入 $2 \mathrm{~mL}$ 一定浓度的供试化合物溶液, 待测药剂至少 4 5 个浓 度, 每个浓度重复 4 次, 设清水为对照. 播种浸种 $4 \sim 6$ $\mathrm{h}$ 的油菜种子 15 粒, $(30 \pm 1){ }^{\circ} \mathrm{C}$ 下, 黑暗培养 $48 \mathrm{~h}$ 后测定 胚胎长度. 与空白对照比较, 计算抑制百分率. 之后采 用 DPS 数据处理系统(8.01 版)数量型数据机值分析法计 算得到 $\mathrm{IC}_{50}$ 值.

\section{References}

[1] Guo, W. C.; Ma, Y.; Li, Y. H.; Wang, S. H.; Li, Z. M. Acta Chim. Sinica 2009, 67, 569 (in Chinese).

(郭万成, 马翼, 李永红, 王素华, 李正名, 化学学报, 2009, 67, 569.)

[2] Xue, Y. L.; Liu, X. H.; Zhang, Y. G. Asian J. Chem. 2012, 24, 1571.

[3] Duggleby, R. G.; Pang, S. S. J. Biochem. Mol. Biol. 2000, 33, 1.

[4] Xue, Y. L.; Zhang, Y. G.; Liu, X. H. Asian J. Chem. 2012, 24, 3016.

[5] Xue, Y. L.; Zhang, Y. G.; Liu, X. H. Asian J. Chem. 2012, 24, 5087.

[6] Wang, J. G.; Ma, N.; Wang, B. L.; Wang, S. H.; Song, H. B.; Li, Z. M. Chin. J. Org. Chem. 2006, 26, 648 (in Chinese).

(王建国, 马宁, 王宝雷, 王素华, 宋海斌, 李正名, 有机化学, 2006, 26, 648.)

[7] Wang, H. X.; Li, F.; Xu, L. P.; Li, Y. H.; Wang, S. H.; Li, Z. M. Chem. J. Chin. Univ. 2010, 31, 64 (in Chinese).

(王红学, 李芳, 许丽萍, 李永红, 王素华, 李正名, 高等学校化 学学报, 2010, 31, 64.)

[8] Chen, P. Q.; Tan, C. X.; Weng, J. Q.; Liu, X. H. Asian J. Chem. 2012, 24, 2808.

[9] Wang, M. Y.; Li, Z. M.; Li, Y. H. Chin. J. Org. Chem. 2010, 30, 877 (in Chinese).

(王美怡, 李正名, 李玉新, 有机化学, 2010, 30, 877.)

[10] Ban, S. R.; Niu, C. W.; Chen, W. B.; Li, Q. S.; Xi, Z. Chin. J. Org. Chem. 2010, 30, 564 (in Chinese).

(班树荣, 牛聪伟, 陈文彬, 李青山, 席真, 有机化学, 2010, 30, 564.)

[11] Wang, M. Y.; Guo, W. C.; Lan, F.; Li, Y. H.; Li, Z. M. Chin. J. Org. Chem. 2008, 28, 649 (in Chinese).

(王美怡, 郭万成, 兰峰, 李永红, 李正名, 有机化学, 2008, 28, 649.)

[12] Liu, X. H.; Tan, C. X.; Weng, J. Q. Phosphorus, Sulfur Silicon Relat. Elem. 2011, 186, 552.

[13] Tan, C. X.; Shi, Y. X.; Weng, J. Q.; Liu, X. H.; Li, B. J.; Zhao, W. G. Lett. Drug Des. Discovery 2012, 9, 431.

[14] Tong, J. Y.; Shi, Y. X.; Liu, X. H.; Sun, N. B.; Li, B. J. Chin. J. Org. Chem. 2012, 32, 2373 (in Chinese).

(童建顷, 石延霞, 刘幸海, 孙娜波, 李宝聚, 有机化学, 2012, 32, 2373.)

[15] Tan, C. X.; Shi, Y. X.; Weng, J. Q.; Liu, X. H.; Li, B. J.; Zhao, W. G. J. Heterocycl. Chem. 2013, DOI: 10.1002/jhet.1656.

[16] Lan, F.; Xu, J. Y.; Li, Y. H.; Wang, S. H.; Li, Z. M. Chem. J. Chin. Univ. 2009, 30, 712 (in Chinese).

(兰峰, 徐俊英, 李永红, 王素华, 李正名, 高等学校化学学报, 2009, 30, 712.)

[17] Wang, L.; Ma, Y.; Liu, X. H.; Li, Y. H.; Song, H. B.; Li, Z. M. Chem. Biol. Drug Des. 2009, 73, 674.

[18] Wang, L.; Tang, M.; Li, W. M.; Li, Y. H.; Wang, S. H.; Li, Z. M. Chem. J. Chin. Univ. 2008, 29, 1371 (in Chinese).

(王雷, 唐蜜, 李文明, 李永红, 王素华, 李正名, 高等学校化学 学报, 2008, 29, 1371.)

[19] Weng, J. Q.; Wang, L.; Liu, X. H. J. Chem. Soc. Pakistan 2012, 34, 1248.

[20] Liu, X. H.; Tan, C. X.; Weng, J. Q. Phosphorus, Sulfur Silicon Relat. Elem. 2011, 186, 558.

[21] Liu, X. F.; Liu, X. H. Acta Crystallogr. 2011, E67, O202. 
[22] Liu, H. J.; Weng, J. Q.; Tan, C. X.; Liu, X. H. Acta Crystallogr. 2011, E67, O1940.

[23] Xu, Q. G.; Liu, T. Y.; Tian, R.; Ma, D. Y.; Li, Q. G. Chin. J. Org. Chem. 2008, 28, 234 (in Chinese).

(徐启贵, 刘天渝, 田睿, 马德银, 李勤耕, 有机化学, 2008, 28, 234.)

[24] Liu, X. H.; Pan, L.; Weng, J. Q.; Tan, C. X.; Li, Y. H.; Wang, B. L.; Li, Z. M. Mol. Diversity 2012, 16, 251 .
[25] Liu, X. H.; Pan, L.; Tan, C. X.; Weng, J. Q.; Wang, B. L.; Li, Z. M. Pestic. Biochem. Phys. 2011, 101, 143.

[26] Liu, X. H.; Zhao, W. G.; Wang, B. L.; Li, Z. M. Res. Chem. Intermed. 2012, 38, 1999.

[27] Liu, X. H.; Pan, L.; Ma, Y.; Weng, J. Q.; Tan, C. X.; Li, Y. H.; Shi, Y. X.; Li, B. J.; Li, Z. M.; Zhang, Y. G. Chem. Biol. Drug Des. 2011, 78, 689 .

(Li, L.; Fan, Y.) 